\title{
Long-lasting growing superhumps in SU UMa stars: QZ Vir
}

\author{
Akira Imada*i \\ Hamburger Sternwarte/Kyoto University \\ E-mail: a_imada@kusastro.kyoto-u.ac.jp \\ Taichi Kato \\ Kyoto University \\ E-mail: tkato@kusastro.kyoto-u.ac.jp \\ Keisuke Isogai \\ Kyoto University \\ E-mail: isogai@kusastro.kyoto-u.ac.jp
}

\section{VSNET Collaboration Team}

We report on the 2015 February-March superoutburst of the well known SU UMa-type dwarf novae QZ Vir (= T Leo). The light curve showed a unique feature in which the precursor was completely separated from the main superoutburst. During the stage between the precursor and main superoutburst, we detected superhumps with the mean period of 0.061181(42) d. Based on the amplitudes and $O-C$ analyses of superhumps, we identified the observed superhump during the faint stage as stage A superhumps. Using the orbital and stage A superhump period of QZ Vir, we derived the mass ratio to be $0.108(3)$. This value indicates that the system is an SU UMatype dwarf novae evolving toward the period minimum. Here we briefly discuss that long-lasting growing superhumps can offer an opportunity to examine the evolutional status of SU UMa-type dwarf novae with moderate orbital periods, and future strategies of photometry of SU UMa-type dwarf novae.

The Golden Age of Cataclysmic Variables and Related Objects IV

11-16 September, 2017

Palermo, Italy

*Speaker.

${ }^{\dagger}$ A footnote may follow. 


\section{Introduction}

SU UMa-type stars are a subclass of dwarf novae. These systems have two types of outbursts: normal outburst and superoutburst. The occurrence of these outbursts depends on the mass transfer rate from the secondary [Osaki(1989)]. During the superoutburst, hump-like modulations, called superhumps, are observed. The light curve of superhumps is understood as tidal dissipation of a precessing eccentric disk (for a review, see [Osaki(1989)]; [Warner(1995)]; [Osaki(1996)]; [Hellier(2001)]).

It is well known that the period of superhumps changes as the superoutburst proceeds. By using extensive photometric data of superhumps, [Kato et al.(2009)] found that the evolution of superhumps consists of three stages: long-constant period (stage A), increasing period (stage B), and short-constant period (stage C). Although the underlying physics of each stage is not clear, it has been gradually accepted that stage A superhump period reflects the dynamical precession rate at the 3:1 resonance radius ([Osaki, Kato(2013)]; [Kato, Osaki(2013)]). The mass ratio of the system can be estimated by using the analytic formulae if the orbital period and stage A superhump period are known (stage A method, [Kato, Osaki(2013)]). Regarding the duration of stage A, [Kato(2015)] showed that the systems with low mass ratios tend to have long duration of stage A. This means that estimating q using stage A superhump period is especially powerful for WZ Sge-type stars, since they show early superhumps and a long duration of stage A. On the other hand, the mass ratio of SU UMa stars are difficult to be determined using stage A method, because of the short duration of stage A or unknown orbital period of the system, or both (the orbital period of SU UMa stars are determined by variations of radial velocity or photometry of eclipes).

QZ Vir is one of the most famous SU UMa-type dwarf novae and the recurrence time of the superoutburst (supercycle) is about a year. The system also shows a normal outburst with the occurrence typically once a year. QZ Vir showed a superoutburst in 2014 May. However, we did not succeeded in obtaining the early stage of the superoutburst, which prevented us from detailed observations of stage A superhumps. In 2015 February-March, QZ Vir showed a superoutburst, during which the system showed a unique light curve: the outburst started with a precursor, after that the magnitude faded almost its quiescent level for a few days, and finally the system entered the main superoutburst-plateau stage. Between the precursor and main plateau stage, superhumps were continuously observed. It turned out that the observed superhumps were indeed stage A superhumps, which enables us to obtain the mass ratio and evolutionary status for the first time. Detail information on the results and discussion have been published in [Imada et al.(2017)].

\section{Results and Discussion}

Figure 1 shows a part of light curve of the 2015 superoutburst of QZ Vir. As can be seen in the figure, the precursor was separated from the main superoutburst. Between the precursor and main superoutburst, we detected superhumps with the mean period of 0.061181(42) d. This value is $\sim 1$ $\%$ longer than that reported in the previous superoutbursts of the same system. In order to identify the stage of the superhumps, we examined the amplitudes and $O-C$ diagram of superhump maxima. Based on these analyses (see figure 4 of [Imada et al.(2017)]), we safely concluded that the observed superhumps between the precursor and main superoutburst were indeed stage A super- 
humps. Using the estimated superhump period and the orbital period of the system, we derived the mass ratio to be $0.108(3)$. The value suggests that QZ Vir is one of the short period SU UMa stars evolving toward the period minimum.

Although the long-lasting superhumps between the precursor and main superoutburst were observed only in a few systems, this phenomenon can offer a possibility that the evolutional status of many SU UMa stars with moderate periods $(P \sim 0.7 \mathrm{~d})$ are clarified. In figure 2 , we present a schematic figure of Patterson diagram. As noted above, WZ Sge stars have a long duration of stage A superhumps and early superhumps. These enable us to precisely determine the evolutional status of WZ Sge stars. Also, period bouncers are much longer duration of stage A and early superhumps. On the other hand, during the plateau stage of the superoutburst, the duration of stage A of SU UMa stars is typically as short as 1 day. This means that we have to determine the mean superhump period of stage A using only $\sim 15$ cycles even if we obtained the whole light curve of stage A superhumps. As a result, stage A method is less effective for SU UMa stars with moderate periods. This is a reason why SU UMa stars with moderate superhump periods have large errors for estimating the mass ratio of the system.

During the 2015 superoutburst of QZ Vir, the faint stage between the precursor and main superoutburst lasted for $\sim 5 \mathrm{~d}$, which means that stage A superhump lasted for $\sim 80$ cycles for QZ Vir. Such a long-lasting stage A superhump can significantly improve our estimation of the period, which enables us to understand the evolutionary status of SU UMa stars with the moderate superhump periods.

In order to detect long-lasting superhumps between the precursor and main superoutburst, we should pay attention not only to a superoutburst, but also to a normal outburst of each system. At present, long-lasting superhumps between the precursor and main superoutburst are hardly observed. Although this is partly due to the possibility that this phenomenon is intrinsically rare, it is obvious that our photometry during the faint stage is lacking. In fact, the AAVSO archival data suggest the presence of growing superhumps between the precursor and main superoutburst in many systems. If the normal outburst occurs near the end of the supercycle, we should continue photometry until the magnitude returns to the quiescent level, in order to see whether or not superhumps appear in a faint stage between the precursor and main superoutburst.

\section{References}

[Hellier(2001)] Hellier, C. 2001, Cataclysmic Variable Stars: How and why they vary (Berlin: Springer-Verlag)

[Imada et al.(2017)] Imada, A., et al. 2017, PASJ, 69, 72

[Kato(2015)] Kato, T. 2015, PASJ, 67, 108

[Kato et al.(2009)] Kato, T., et al. 2009, PASJ, 61, S395

[Kato, Osaki(2013)] Kato, T., \& Osaki, Y. 2013, PASJ, 65, 115

[Osaki(1989)] Osaki, Y. 1989, PASJ, 41, 1005

[Osaki(1996)] Osaki, Y. 1996, PASP, 108, 39

[Osaki, Kato(2013)] Osaki, Y., \& Kato, T. 2013, PASJ, 65, 95 


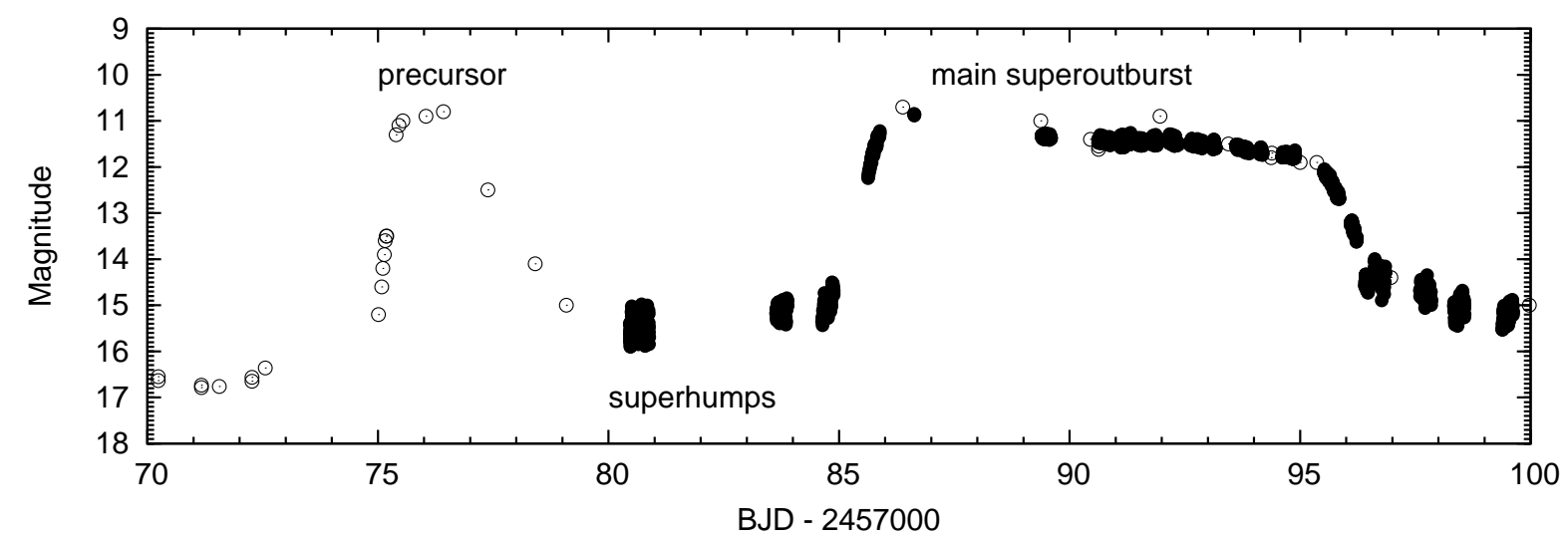

Figure 1: A part of the light curve during the 2015 superoutburst. Black points indicate CCD observations, while white points indicates visual observations posted to the AAVSO. During the faint stage between the precursor and main superoutburst, superhumps were observed with the mean period of $0.061181 \mathrm{~d}$.

[Warner(1995)] Warner, B. 1995, Cataclysmic Variable Stars (Cambridge: Cambridge University Press)

\section{DISCUSSION}

Elena Pavlenko We should not start observations after normal outburst and we should not stop observations during normal outburst!

Akira Imada You are right! We should especially focus on normal outburst near the end of supercycle. Such a normal outburst may be a precursor.

Nataly Katysheva What is a differ between normal outburst and precursor ?

Akira Imada The physics of outburst is basically the same (I mean the disk instability). But the precursor triggers a superoutburst. Like the present observations, superhumps appear near the end of the precursor.

Kenji Tanabe You mentioned Kyoto 3.8m telescope is soon coming. But my recent information is that the secondary mirror is not yet made and the primary is partial. What does "soon" mean?

Akira Imada I am not a core member of Kyoto group so that I cannot comment on when the telescope starts. But Kyoto $3.8 \mathrm{~m}$ telescope will concentrate on dwarf novae and we hope improvement on dwarf novae research using the telescope. 


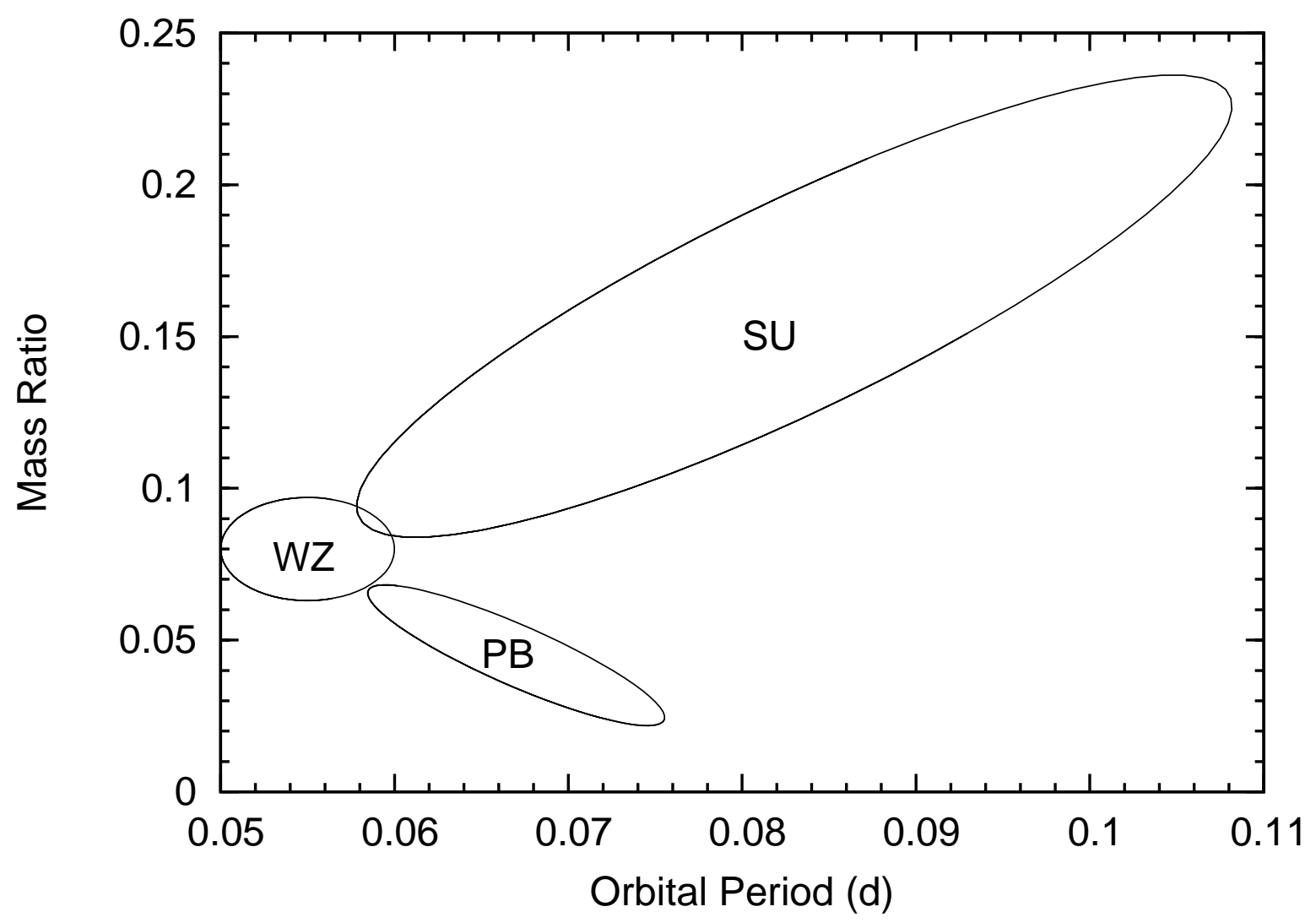

Figure 2: Schematic diagram of evolutionary status of SU UMa stars. The x axis denotes the orbital period and $y$ axis denotes the mass ratio of the system. We can determine the locations of WZ Sge stars (WZ) and period bouncers (PB) if early and stage A superhumps are detected. On the other hand, the location of SU UMa stars (SU) are difficult to be determined because of the short duration of stage A and unknown orbital period (We also should perform radial velocity studies using a 3-4m class telescope for SU UMa stars with unknown orbital periods.). 